\title{
CONCEPCIONES DE LOS ESTUDIANTES DE EDUCACIÓN BÁSICA SOBRE ECOSISTEMA. UNA REVISIÓN DOCUMENTAL
}

\section{BASIC EDUCATIION STUDENTS` CONCEPTIONS ABOUT ECOSYSTEM. A DOCUMENTARY REVIEW}

\section{Por: María Eugenia Rincón $\mathbf{H}^{2}$}

Recibido:18-08-2011

Aceptado:23-09-2011

\section{RESUMEN}

En el campo de la ecología y en general en el área de ciencias naturales y educación ambiental, el concepto de ecosistema y conceptos relacionados con su dinámica como flujos de energía, ciclos de nutrientes, redes tróficas, interacciones, estabilidad y equilibrio y efecto de las perturbaciones sobre la dinámica del ecosistema son fundamentales; sin embargo en los estudios realizados acerca de las concepciones sobre la noción de ecosistema se han encontrado una serie de dificultades y concepciones erróneas respecto a este concepto, en diferentes niveles de escolaridad. El propósito de este trabajo es presentar una revisión documental sobre las concepciones que tienen los estudiantes de educación básica sobre ecosistema y las perspectivas de abordaje para el análisis de las mismas en estos estudios.

Palabras claves: Concepciones, ecosistema, cognición causal, cambio conceptual, modelización, hipótesis de progresión.

\section{ABSTRACT}

In ecology field and in general in Natural Science and Environmental Education area, the concept of ecosystem and other concepts related to its dynamics such as energy flows, nutrient cycles, food webs, interactions, stability and balance and effect of disturbances on ecosystem dynamics are fundamental. However, in conducted studies on conceptions of ecosystem, a series of difficulties and misconceptions about this concept were found at different levels of schooling. The objective of this study was to present a literature review about student's conceptions of ecosystem and the perspectives to analyze these ideas.

Key words: conceptions, ecosystem concept, Causal cognition, conceptual change, modelization, progression hypothesis.

\section{LOS ESTUDIOS SOBRE LAS CONCEPCIONES ACERCA DEL ECOSISTEMA}

Las investigaciones acerca de las concepciones sobre ecosistema se han abordado como se mostrará a continuación principalmente desde cuatro perspectivas: cognición causal, cambio conceptual, modelización e hipótesis de progresión. En esta revisión, se hará énfasis en las concepciones de los estudiantes de educación básica.

\footnotetext{
${ }^{1}$ Este documento hace parte de los desarrollos de la tesis Doctoral "Conocimiento didáctico del contenido sobre ecosistema en formadores de formadores de programas de licenciatura en Biología. Director Edgar Valbuena. Doctorado interinstitucional en Educación. Universidad Pedagógica Nacional.

${ }^{2}$ Docente Departamento de Biología. Universidad Pedagógica Nacional. merincon001@hotmail.com
} 


\section{Estudios basados en la perspectiva de la cognición causal}

En cuanto a las investigaciones relacionadas con cognición causal, se han llevado a cabo principalmente con estudiantes de primaria y secundaria. Estos estudios han permitido desarrollar el razonamiento causal y los juicios causales y su relación con muchos dominios cognitivos. La mayoría de las investigaciones se han centrado en juicios acerca de relaciones simples de causa - efecto. En realidad las relaciones causales no son aisladas de otras sino que tienden a estar relacionadas en sistemas dinámicos (Grotzer, 2009).

La Cognición causal involucra no solo identificar la causa de algún efecto, sino también integrar colecciones de eventos individuales dentro de una representación organizada de cadenas y redes de relaciones causales; en este campo las investigaciones se han enfocado principalmente en indagar acerca de de la interdependencia de los organismos en los ecosistemas, relaciones entre organismos en la red trófica, y solo algunos estudios han abordado el ciclaje de la materia y los efectos de las perturbaciones en la red trófica.

Las dificultades más frecuentemente encontradas por los diversos autores son:

- Centramiento en lo evidente y lo próximo a la experiencia de los estudiantes, destacan más la importancia de los animales que de las plantas (Bell- Basca, Grotzer, Donis, \& Shaw, 2000). La prevalencia dada al reino animal y para el caso de las representaciones graficas (animales domésticos) se debe principalmente al carácter utilitario que los estudiantes le dan a los mismos (Rincón, Medellin, \& Vargas, 2004).

- Dificultades al razonar acerca de la dimensión espacial en los ecosistemas, ellos tienden a razonar localmente y no incluyen escalas mayores y menores (BellBasca et al. 2000; White 2000).

- En cuanto a las interacciones entre los componentes del ecosistema, la mayoría de los estudiantes destacan las relacionadas con la alimentación y unas pocas con el hábitat. En las relaciones tróficas mencionan especialmente la relación depredador - presa. Igualmente, se consideran más las relaciones antagónicas que las de cooperación (Leach, Driver, Scott, \& Wood-Robinson, 1996a). De otra parte, los componentes abióticos no son muy tenidos en cuenta y difícilmente se establecen interacciones con los componentes bióticos (White, 1997 y 2000).

- Algunos estudiantes piensan que los carnívoros podrían existir solamente si sus presas se reprodujeran abundantemente, sin considerar la relación de estas con su fuente de alimento (Leach, Driver, Scott, \& Wood-Robinson, 1992).

- En relación a los flujos de energía, muchos niños expresan la idea de que las plantas elaboran el alimento solo para el beneficio de los animales y la gente y no que este sea esencial para las plantas mismas. Los niños no reconocen que la fotosíntesis es el proceso mediante el cual la energía del ambiente llega a estar disponible para las plantas y posteriormente para los animales (Leach, et al. 1992) y no reconocen la importancia de la energía para los procesos de crecimiento y desarrollo de las plantas (Driver, 1994 ). Varios consideran la luz como "alimento" para las plantas o como un reactivo para la fotosíntesis y la mayoría de estudiantes no entienden las transferencias de energía entre los organismos. Igualmente, Gayford (1986) reporta que estudiantes adolescentes entre 17 y 18 años consideran que el flujo de energía es transportado de un lugar a otro en los sistemas biológicos y que puede ser almacenado como un material. 
- La valoración del papel de cada elemento en el ecosistema, se plantea frecuentemente desde la perspectiva teleológica y antropológica en niños entre 5 y 7 años y este razonamiento fue poco común en niños mayores de 9 años (Leach, et al. 1992). Sin embargo, Driver (1994), plantea que este tipo de razonamiento también se da en estudiantes entre 12 y 13 años, quienes utilizan un razonamiento teleológico relacionado con los animales y las redes alimentarias, postulando que las plantas producen alimentos en beneficio de los animales y las personas, o que los demás animales existen para el beneficio de los seres humanos. Se da más importancia a los consumidores, principalmente a los depredadores (Leach, Driver, Scott, \& Wood-Robinson, 1996b).

- Las interacciones que se abordan entre los componentes bióticos, se dan más a nivel de individuo que de población (Leach et al. 1996b; Grotzer \& Basca 2003; Griffiths \& Grant, 1985), lo que se dificulta razonar acerca de los controles y el equilibrio en el sistema.

- Respecto a la progresión en el razonamiento acerca de los fenómenos ecológicos, de acuerdo a Leach, et al. (1992), los niños más jóvenes entre 5 y 7 años, tienden a pensar solo en términos de los organismos individuales que la gente mantiene (mascotas, animales del zoológico, plantas de la casa) y los cuales necesitan los humanos para su supervivencia. Los niños mayores entre (7-11 años) amplían sus descripciones a organismos silvestres como individuos y no como población, a pesar de que algunos de ellos consideran que son alimentados y cuidados por las personas. La mayoría de los estudiantes mayores de 13 años, involucran el concepto de población silvestre, pero sus explicaciones son principalmente descripciones de tipo naturalístico.

- En general los estudiantes tienen dificultad de razonar acerca del ecosistema como un sistema (pensamiento sistémico) y generalmente representan las redes tróficas como secuencias lineares siguiendo un patrón de causalidad lineal, basado en una relación de causa- efecto, la cual es unidireccional y directa (Driver, 1994; Leach et al. 1996b; White, 1997; Grotzer, 2009). Con frecuencia tanto estudiantes de primaria como secundaria no tienen en cuenta la causalidad tipo domino (Barman, Griffiths, \& Okebukola, 1995; Grotzer \& Bell- Basca ,2003), donde el efecto se propaga desde la causa (perturbación) en patrones tipo domino, en este patrón ya se reconoce que si faltan los productores esto afectaría no solamente a los consumidores primarios sino también al resto de consumidores (secundarios, terciarios etc.).

Los autores en mención plantean que entender y razonar adecuadamente acerca de los ecosistemas significa entender un número de diferentes tipos de patrones causales más complejos; estos incluyen según Grotzer (2002): 1. Patrones tipo domino, son importantes para entender por ejemplo el flujo de energía en las redes alimentarias; 2. Patrones causales cíclicos, en los cuales una causa desencadena un efecto que en últimas tiene un efecto sobre la causa inicial. Son importantes para entender los ciclos biogeoquímicos y 3. Causalidad en doble vía, en el cual un evento o relación tiene efectos mutuos y con frecuencia simultáneos. Cada componente tiene un efecto sobre el otro, por lo que cada uno actúa como un efecto y una causa. Puede aplicarse a una relación simbiótica como una abeja polinizando una flor), donde un evento o acción, afecta a ambos organismos. La comprensión de la causalidad compleja es una habilidad fundamental para el aprendizaje de los ecosistemas. Sin embargo, incluso después de la instrucción de una unidad didáctica, los estudiantes tienen considerables dificultades de 
razonamiento acerca de las estructuras y relaciones complejas que se dan en los ecosistemas.

-En (1997), White encontró un efecto de disipación en los juicios que planteaban los alumnos de secundaria acerca del efecto de una perturbación en un determinado nivel de una red trófica, según el cual, las consecuencias de una perturbación, cuyo efecto se daba en un eslabón especifico, tendía a debilitarse o disiparse a medida que se alejaba del foco de perturbación, homologando este comportamiento con el de los sistemas físicos, es decir, que solo se afectaría la población directamente relacionada y el cambio en una población ocasionado por una perturbación, no sería pasado a lo largo de diferentes vías de la red trófica. Similares resultados fueron reportados por Grotzer, (2009) y Griffiths \& Grant, (1985).

En este sentido, White (2000) encontró que los juicios de los estudiantes cambiaban cuando observaban en una representación gráfica de una red trófica que había una conexión directa, argumentaban que los efectos eran superiores, mientras que cuando había una ramificación el efecto era menor. Igualmente, el juicio del efecto es mayor cuando se encuentra en una posición terminal que en una posición intermedia (porque son puntos que tienen generalmente una sola conexión). Estos hallazgos pueden ser explicados como efectos de un modelo mental que manejan los estudiantes de influencia y resistencia, como sucede en los sistemas físicos.

En cuanto a los ciclos de la materia Smith \& Anderson (1986) encontraron que la mayoría de los estudiantes de 12 años de su muestra, consideraban que alguna clase de proceso cíclico tenía lugar en los ecosistemas; sin embargo, la mayoría de ellos pensaba en términos de eventos de causa- efecto, con la materia siendo creada o destruida en estos eventos y luego la secuencia iniciaba de nuevo. Algunos de ellos consideraban una forma de reciclaje a través de los materiales del suelo, pero no incorporaban el agua, el oxígeno y el dióxido de carbono como parte de los ciclos. Ellos no establecían conexiones entre los ciclos de la materia y otros procesos que involucraban la producción, consumo y utilización del alimento. Aún después de la instrucción de estos contenidos hubo pocos cambios solo un $4 \%$ de los estudiantes lograron comprender las transformaciones de la materia orgánica y la relación entre componentes bióticos y abióticos en los ciclos de la materia (Leach et al. 1992 y Leach et al. 1996a).

Teniendo en cuenta lo anterior, varios autores coinciden en que no se reconocen el papel de los microorganismos en los procesos de descomposición de la materia orgánica y que generalmente se asocian estos procesos más a animales pequeños que a los microorganismos (Leach et al. 1996a; Grotzer \& Bell- Basca ,2003, Grotzer, 2009). En cuanto a los procesos de descomposición de la materia orgánica, los alumnos de mayor edad no tiene en cuenta en sus explicaciones los procesos químicos que ocurren, sino que plantean su función solo en términos de fuente de alimento para otros organismos (Leach et al 1996b); sin embargo, BellBasca et al. (2000), encontraron que solo algunos estudiantes de secundaria a través de actividades de discusión, mostraron una mayor comprensión de los procesos de descomposición y los mecanismos que la causan.

\section{Estudios desde la perspectiva del cambio conceptual}

Otro referente teórico desde el cual se han abordado las concepciones acerca del ecosistema es desde el cambio conceptual. Según Ibarra Murillo \& Gil Quiléz (2005), esta perspectiva abarca la búsqueda de modelos, estructuras y 
conceptos que son comunes a las diferentes ciencias experimentales. Esta implica, el paso del conocimiento cotidiano al conocimiento científico requiere una reestructuración en los niveles ontológicos, epistemológicos y conceptuales. Marín \& Cárdenas (2011), consideran que "el modelo de cambio conceptual está basado en la analogía "el alumno como científico" y en su primera fase, busca debilitar las ideas previas de los alumnos haciéndolas entrar en conflicto cognitivo con evidencias empíricas o argumentos teóricos y, en una segunda fase, se presentan los conceptos correctos de ciencias como ideas que son más plausibles y útiles para explicar tales evidencias y argumentos" (p.40).

Desde esta perspectiva, algunas investigaciones se han centrado en determinar cambios específicos en la comprensión conceptual que tienen estudiantes de básica secundaria, acerca de las relaciones alimentarias en un ecosistema y conocer los obstáculos básicos que podrían dificultar una comprensión más profunda de esta temática. Para entender la complejidad de las interacciones alimentarias Eilam (2002), propone cuatro dimensiones: 1. Dimensión de macronivel de las relaciones tróficas, que involucra los componentes, sus relaciones específicas y el orden de cada componente en la cadena alimentaria; 2. Dimensión de micronivel, la cual incluye los procesos moleculares y microprocesos de transferencia de materia y energía en los ecosistemas; 3. La dimensión espacial de los ecosistemas, en relación con ocurrencias simultáneas de procesos en el espacio y los múltiples roles tróficos de sus componentes bióticos; 4. La dimensión temporal, relacionada con las fuerzas evolutivas que actúan a largo plazo en los ecosistemas y que afectan sus relaciones tróficas.

El autor en mención, señala tres dificultades relacionadas principalmente con la dimensión espacial, encontradas en una muestra de estudiantes de secundaria en Israel: 1. La incapacidad de comprender ocurrencias múltiples como simultáneas (por ejemplo, la pérdida de una población en un ecosistema puede afectar a otras al mismo tiempo); 2. No considerar que cada elemento de la cadena alimenticia puede ocupar más de un nivel trófico (un animal puede ser un consumidor primario en una cadena alimenticia y un consumidor secundario en otra); 3 . Tener una percepción unidireccional de las relaciones tróficas.

Otras dificultades planteadas por Eilam (2002), tanto para el grupo control, como para el grupo experimental en los pretest realizados, coinciden con algunos de los resultados descritos por otros autores, así: las relaciones alimentarias presentan patrones unidireccionales o cíclicos entre depredadores y presas (Leach, Driver, Scott, \& Wood-Robinson, 1991; Fernández \& Casal, 1995; Soylu, 2006; Magntorn \& Hellden 2007). Fernández \& Casal (1995), encontraron que los niveles más ampliamente reconocidos por los estudiantes de secundaria son los herbívoros y los carnívoros. En el mismo sentido, Çetin, (2003) (citado en Çetin, 2007) reporto que estudiantes turcos de noveno grado de secundaria planteaban que los consumidores secundarios constituyen el primer eslabón para la cadena alimenticia y que los alimentos producidos eran tomados por los consumidores terciarios que eran animales. El nivel de los productores sólo se menciona en los casos en los que hay una completa identificación de niveles, lo cual indica la poca importancia que se asigna a los vegetales en el mantenimiento de la vida. Esta idea viene a confirmar los resultados de otros estudios que señalan la falta de identificación de los vegetales con su función como productores de la mayor parte de la materia orgánica de los ecosistemas.

Otra concepción derivada es que los estudiantes identifican cada eslabón o nivel trófico con individuo y no con población. En tales situaciones los alumnos expresan 
la idea de catastrofismo asociado a la depredación. Peterfalvi, Rumelhard, \& Verin (1987), señalan que las figuras y los ejemplos con que se suelen representar las cadenas tróficas en los textos refuerzan la idea de fragilidad de la cadena y de sus componentes.

Soylu (2006), encontró en una muestra de 600 estudiantes turcos de grado octavo entre 13 y 16 años, varias dificultades en el aprendizaje de algunas nociones ecológicas como: cadena alimentaria, red trófica, flujo de energía y la fuente de energía. Por ejemplo, la mayoría de los estudiantes dibujan las cadenas alimenticias como cíclicas, no lineares y ubican a los descomponedores en la parte superior de la pirámide de energía y argumentan que dicha posición se debe a que estos descomponen a todos los organismos de los niveles inferiores, lo cual indica que no se establecen diferencias entre los diversos tipos de pirámides (de números, biomasa y energética). Resultados similares fueron registrados por Adeniyi (1985), Griffiths \& Grant (1985) y Eilam (2002). Ellos plantean que los estudiantes consideran que los animales que están en los niveles superiores se alimentan de todos los que están en los niveles tróficos anteriores y por esta razón tienen más energía. En el año 1985, Adeniyi reporto que estudiantes Nigerianos entre 13 y 15 años consideran que la energía se va adicionando, así que el hombre al obtener energía tanto de la vaca como de las plantas, tiene más energía.

Otra concepción muy arraigada en los estudiantes acerca de la red trófica en estos estudios, es que una población solo se vería afectada si esta se relaciona directamente con otra, como en el caso de la relación depredador- presa (Soylu, 2006; Eilam, 2002; Çetin, 2007).

También el concepto de descomponedor se muestra muy alejado de su acepción ecológica. Los ejemplos propuestos por los estudiantes inducen a pensar que los descomponedores son seres o sustancias dañinas que perjudican al conjunto de la cadena (Fernández \& Casal, 1995 y Çetin, 2007).

Respecto a los ciclos de la materia, los estudiantes no tienen claridad acerca de lo que es un ciclo y las transformaciones que en el ocurren a pesar de que estos procesos se incluyen cuando se describen las propiedades de los ecosistemas (Magntorn y Helldén, 2007 y Çetin, 2003 (citado en Çetin 2007)). Igualmente no establecen ninguna relación cíclica entre productores, consumidores y descomponedores. Por ejemplo, los estudiantes no pueden entrever en el ciclo del carbono, la relación entre los distintos organismos y compartimentos abióticos dentro de un ecosistema. Magntorn y Helldén, (2007), sugieren que la descomposición es un concepto más intuitivo que la fotosíntesis y constituye un concepto clave para ayudar a los estudiantes de secundaria a comprender los procesos cíclicos en los ecosistemas.

Otra dificultad generalizada en las concepciones de los estudiantes de secundaria es que los componentes abióticos no son muy tenidos en cuenta y difícilmente se establecen interacciones con los componentes bióticos (Eilam, 2002). De manera similar, Lin \& Hu (2003), encontraron que en los mapas conceptuales que elaboraron estudiantes de grado séptimo de 13 años de edad en Taipei, no establecieron este tipo de interacciones entre estos componentes. De acuerdo a Magntorn (2007), estos sistemas de razonamiento que involucran la interacción entre los factores bióticos y abióticos son difíciles, y los estudiantes a menudo se refieren únicamente a relaciones causales entre los componentes bióticos, vinculando al nivel más cercano hacia arriba o hacia abajo en el ecosistema (por 
ejemplo, cómo las plantas se ven afectados por el número de herbívoros, pero no por el nivel de los carnívoros).

Según Fernández \& Casal (1995), el aspecto más notable del valor atribuido a esta interdependencia es que las sustancias o componentes del entorno (agua, luz, etc.) no son consideradas con carácter limitante, ni tampoco perturbadas o modificadas por influencia de los factores bióticos, incluida la especie humana. Generalmente, los estudiantes de bachillerato estiman que ciertas sustancias, como el agua, la luz del sol, son imprescindibles para la vida. Sin embargo, no se relaciona su importancia con la intervención de dichos componentes en la formación de estructuras de los seres vivos.

De otra parte, distintos investigadores coinciden en que durante la intervención las estrategias de intercambio diseñadas, como involucrar al grupo experimental en salidas de campo y en proyectos de investigación, han contribuido con la evolución de las ideas previas de los y las estudiantes hacia nociones más adecuadas desde el punto de vista científico. Eilam (2002), plantea que muchos de sus estudiantes pudieron aplicar parte de su conocimiento a otros ecosistemas a una escala mayor. "La selección de su propia investigación y la autoregulación de su aprendizaje promovieron el interés y la motivación de de los estudiantes para participar en este proceso a largo plazo" (p.663). (Fernández \& Casal 1995, señalan que después de la intervención, los estudiantes que realizan el trabajo de campo (grupo experimental) expresan de forma significativamente mejor la organización de los conceptos de ecología, reconocen mayor cantidad de componentes y adquieren mejor comprensión de las relaciones, probablemente porque tienen un referente en el que situar los análisis que se van realizando en el aula o laboratorio. El seguimiento del ciclo de los elementos y la importancia de los mismos muestran diferencias significativas entre los dos grupos. También, los estudiantes del grupo experimental establecen mayor número de conexiones entre los componente del ecosistema, mediante explicaciones de la relación entre unos y otros componentes.

En este sentido, Magntorn (2007), también destacan la importancia de las salidas de campo como una estrategia para lograr aprendizajes significativos y argumentan que este tipo de prácticas en un ecosistema específico (bosque) ayuda a los estudiantes a establecer las interacciones entre los seres vivos y el medio. Después de estas prácticas, los estudiantes no solo definían un mayor número de componentes y relaciones tróficas, sino también establecían interacciones entre los descomponedores, las plantas y el suelo. Igualmente, dicha estrategia favorece la transferencia de conocimientos a otros tipos de ecosistemas (estanque), especialmente en términos de grupos funcionales.

Sin embargo, algunos autores señalan que a pesar de que las estrategias de intercambio diseñadas para promover cambios conceptuales han sido valiosas a la hora de producir la evolución de las ideas previas de los estudiantes hacia nociones más adecuadas desde el punto de vista científico, se presentan dificultades en la comprensión de temas concernientes con la energía, evolución y la complejidad del ecosistema (Eilam, 2002; Magntorn y Helden, 2007). Al respecto, diversos autores coinciden en que algunas nociones ecológicas relacionadas con los ecosistemas, son persistentes al cambio y dificultan el aprendizaje (Adeniyi, 1985; Çetin, 2003; Eilam, 2002; Gallegos, Jerezano, \& Flores, 1994; Griffiths \& Grant, 1985 y Reiner \& Eilam, 2001).

Una de las principales dificultades, está relacionada con la predicción de cambios en la dinámica de los ecosistemas después de una perturbación. Algunos estudios 
han identificado los esquemas conceptuales que los alumnos de secundaria y de la ESO en España, utilizan para interpretar los cambios en los ecosistemas después de una perturbación y analizar de qué forma utilizan el concepto de la sucesión ecológica para interpretar estos procesos (Ibarra Murillo \& Gil Quiléz, 2005 y 2009). Carlson (2002), sugiere que la noción de transformación es clave para el desarrollo de la comprensión ecológica de los cambios sucesionales en la dinámica del ecosistema. El concepto de sucesión es difícil de aprehender, ya que está fuertemente influenciado por la idea de equilibrio de la naturaleza.

Al respecto algunas de las dificultades planteadas por algunos autores son:

- Predomina una idea de equilibrio estático entendido como un óptimo, como homogeneidad, siendo sólo las fluctuaciones reversibles en torno a un estado de equilibrio, superpuestas en el desarrollo del ecosistema hacia un clímax preestablecido mediante la sucesión ecológica clímax (Ibarra Murillo \& Gil Quiléz, 2005). Lo anterior proporciona una visión limitada de la dinámica de un ecosistema. Igualmente, Magtorn (2007), encontró que la mayoría de los estudiantes de secundaria, consideran que el paisaje no cambia en absoluto a través del tiempo, mientras que solo una escasa muestra de estudiantes manifiesta que los cambios en los ecosistemas son graduales, pero no mencionan el impacto humano. Solamente un estudiante en la primera entrevista expresó la idea de que los ecosistemas no son estables y cambian con el tiempo, y que los seres humanos suelen tener un impacto sobre estos.

- En el plano ontológico, de acuerdo con Ibarra Murillo \& Gil Quiléz (2009), los cambios sucesionales no tienen para los alumnos la categoría ontológica de proceso, sino representan un "evento" o suceso que tiene una etapa inicial y una final. Por lo que cuando se trata de predecir los cambios en los ecosistemas a largo plazo, destaca el carácter interpretativo finalista, acausal y principalmente cualitativo que hacen los alumnos sobre los cambios sucesionales. En definitiva, las relaciones entre los seres vivos y entre éstos y el ambiente no parecen ser necesarias para explicar o describir los cambios en la naturaleza. Sin embargo, la interpretación de los fenómenos ecológicos en clave de interacciones es fundamental para el conocimiento de los ecosistemas, y la dificultad radica en explicitar estas relaciones e incluir en ellas también las acciones humanas (Jacobson, 2000).

- Respecto a las bases epistemológicas que sustentan las ideas de los alumnos sobre la sucesión, Ibarra Murillo \& Gil Quiléz (2009) concluyen que las ideas vitalistas principalmente, aunque también las teleológicas y las antropomórficas, emergen con fuerza en los discursos de los alumnos de secundaria y parecen ser suficientes para ellos, a la hora de explicar y predecir los cambios en la naturaleza.

- En un estudio realizado por Ibarra Murillo \& Gil Quitéz, (2005), en relación con la transposición didáctica de la teoría de la sucesión desde la ciencia hasta los libros de texto de secundaria, encontraron que en la mayoría de los textos, la ecología no es sólo presentada como la ciencia del equilibrio sino que, además, la idea de equilibrio de la naturaleza se presenta como un axioma en lugar de hipótesis que ha de ser verificada. Los libros de texto muestran la ecología mayoritariamente desde el paradigma holista o ecosistémico y así mismo refuerzan la idea del equilibrio ecológico y el determinismo de los cambios en los ecosistemas. Y señalan además, al igual que otros autores (Magro, Simonneaux, Favre, \& Hemptinne, 2002) que el pensamiento científico de los profesores, que enseñan ecología también está muy mediatizado por este pensamiento. 


\section{Estudios basados en la modelización}

Otras investigaciones se han enfocado en evaluar el efecto de una intervención didáctica en el aprendizaje de la noción de ecosistema mediante procesos de modelización, cuyo propósito ha sido propiciar el desarrollo de un pensamiento sistémico en los estudiantes. Según Peisajovich (2005), dentro de los planes escolares el concepto de ecosistema, al igual que muchos otros contenidos en ciencias naturales son tratados desde una perspectiva analítica, esto es, entendiendo cada parte del objeto por separado para formar un panorama general luego. Esta perspectiva se muestra inadecuada para lograr un entendimiento de las dinámicas involucradas en un ecosistema en su verdadera magnitud, naturaleza y complejidad. Peisajovich propone que el estudio de los ecosistemas se realice teniendo en cuenta su verdadera naturaleza sistémica y la complejidad de las relaciones que se dan entre sus elementos.

La utilización de procesos de modelización en la enseñanza de las ciencias es una propuesta relativamente reciente que responde a la necesidad de apoyar la enseñanza de las ciencias sobre un referente metodológico claro. Es una perspectiva que a principios de esta década toma fuerza con estudios sobre temas relacionados que sirvieron como sus precursores tales como modelos científicos en la enseñanza, simulación, analogías y razonamiento por medio de modelos (Grajales \& González, 2009). Las simulaciones son una herramienta importante en cualquier esfuerzo para desarrollar el pensamiento sistémico, ya que tienen el potencial de poner de relieve el carácter dinámico de los sistemas.

En el año 2008, Westra analizo en una población de estudiantes de educación secundaria en Holanda, la influencia de procesos de modelización y autenticas prácticas ecológicas, en la comprensión de la dinámica y la complejidad de los ecosistemas y en el desarrollo de un pensamiento sistémico. Los resultados indican que la mayoría de los estudiantes reconocen bien los diferentes niveles de organización abordados como ecosistema, comunidad, población, organismo y establecen relaciones con el medio abiótico. Sin embargo, muchos estudiantes parecen tener problemas en: 1.la comprensión compleja y dinámica del ecosistema, especialmente en ejemplificar la relación entre complejidad, dinámica, estabilidad y diversidad en un ecosistema, el autor plantea que esto podría deberse a que la perspectiva cibernética es la que predomina en los estudiantes, los profesores y los textos de secundaria, por lo que persiste en los estudiantes después de la intervención una idea de equilibrio estático entendido como un óptimo, con fluctuaciones reversibles en torno a un estado de equilibrio. Estas dificultades han sido planteadas también por Ibarra Murillo \& Gil Quiléz (2009) en el apartado anterior; 2. Reconocer un ecosistema como un sistema abierto, lo que significa que no sólo las partes, sino que las relaciones entre las partes son fundamentales en su organización y que los límites de los sistemas no son siempre claros y especialmente; 3 . la construcción autónoma de los modelos informáticos a partir de los datos de sus prácticas, lo que podría ser causado por no entender bien la estructura abstracta del modelo, donde los estudiantes no ven la relación entre los componentes de esta estructura y los fenómenos naturales.

Algunas de las anteriores dificultades también han sido planteadas por Labbe (2002), quien analizo las concepciones relacionadas con el ciclo de la materia y específicamente con el ciclo del carbono en estudiantes de octavo grado provenientes de diferentes liceos, pues considera que el ciclo del carbono constituye un concepto complejo dado que se relaciona con otros conceptos como 
la fotosíntesis, la respiración y la descomposición. Las concepciones se analizaron teniendo en cuenta la diversidad de componentes involucrados en un ciclo, la noción de ciclo, las formas en que está presente el carbono y su localización, los mecanismos de transferencia y el tiempo de referencia. Entre las dificultades encontradas, los autores mencionan que: 1. la mayoría de los estudiantes no tuvieron en cuenta los datos cuantitativos obtenidos en sus prácticas, lo cual constituye una limitación importante para los modelos así construidos; 2 . una visión homogénea de los depósitos de carbono y el desconocimiento de las diferentes formas de carbono; 3. no considerar todas las transformaciones químicas, las cuales son un componente esencial de los mecanismos explicativos del ciclo del carbono y la no aplicación de los fundamentos de la química dentro de los contextos biológicos parece ser una dificultad previsible para la comprensión de estos procesos y; 4. los estudiantes no consideraron las diferentes escalas de tiempo de formación y acciones de movilización, lo cual influye a su vez en la poca predictibilidad de los modelos planteados.

En Colombia, Grajales \& González (2009) evaluaron en un grupo de estudiantes de séptimo grado de La Normal Superior de Medellín, los efectos de la aplicación de procesos de modelización, en la enseñanza y aprendizaje de contenidos relacionados con las ciencias naturales desde una perspectiva sistémica. Esta intervención se llevo a cabo en el contexto de una unidad didáctica sobre el concepto de ecosistema con la cual se pretendía que los estudiantes desarrollaran una visión más compleja y profunda de las dinámicas implicadas en el funcionamiento de este sistema. Antes de la intervención tanto en el grupo control como en el experimental, persistía una visión lineal y mecánica del funcionamiento de los ecosistemas representados en los modelos. Luego de la intervención los estudiantes fueron capaces de identificar relaciones de flujo de energía, competencia, comunicación y sostenibilidad entre otras, generando abstracciones teóricas a partir del objeto real. Los estudiantes del grupo experimental expresaron en sus representaciones del ecosistema un entramado de relaciones más complejo, tanto en número como en la naturaleza y características de dichas relaciones, en contraste con su percepción inicial sobre los ecosistemas, que en la mayoría de los casos se reducía a relaciones lineales de transferencia de materia por medio de la alimentación. Además entre ellos primaba la idea de que los seres humanos ocupan un papel privilegiado con respecto a los demás organismos.

Resultados similares fueron encontrados por Evagoru, Korfiatis, Nicolaou, \& Constantinou (2009), en niños de primaria de 5 y 6 grado, quienes consideran que el ambiente de aprendizaje propuesto, el cual incluía simulaciones interactivas de un ecosistema de marisma, fomento considerables mejoras en las habilidades de pensamiento sistémico durante un proceso relativamente breve de aprendizaje. Sin embargo, los autores plantean que el ambiente de aprendizaje no tuvo éxito en los procesos de retroalimentación, dadas las dificultades encontradas por los estudiantes.

\section{Estudios basados en la perspectiva de la hipótesis de progresión}

A diferencia de los trabajos anteriores enmarcados en las perspectivas de la cognición causal, el cambio conceptual y la modelización, los estudios que se mencionan a continuación están enmarcados en la perspectiva de la Hipótesis de Progresión, la cual constituye un marco de referencia para la construcción del conocimiento, guiando la organización y secuenciación de los contenidos (García \& Rivero, 1996; García, 1997). 
También supone un cierto gradiente de complejidad (García, 1995b), con diferentes niveles de formulación (Astolfi, 1988) que se refieren, por una parte, a los sucesivos estados por los que pasa un individuo en la evolución de sus ideas en los diferentes momentos en el desarrollo de determinadas estructuras cognitivas, las dificultades de aprendizaje a superar para que la progresión sea posible y por otra, a las diferentes etapas que se programan para la construcción del contenido -los posibles constructos intermedios, las aproximaciones sucesivas a una noción (García, 1997). Es por ello que los niveles de formulación son objetivos a largo plazo.

Astolfi (2001), plantea que los enunciados producidos en el curso de los aprendizajes científicos, en relación con una misma idea o al término de las actividades de estructuración, son muy variados y dependen a la vez del nivel de escolaridad considerado y de la naturaleza del problema científico al que responda cada uno. Esta variedad de enunciados posibles conduce a determinar los niveles de formulación. Así, señala como ejemplo, que en clase de biología de $6^{\circ}$ se pueden construir diversas formulaciones de la idea de régimen alimentario, a partir de observaciones de animales domésticos, así como de experiencias sencillas y de documentos, pero después de que los estudiantes hayan estudiado las redes tróficas, la idea de régimen alimenticio podrá reformularse de otra manera y en otro nivel.

En una investigación realizada por García (1995a), con alumnos adolecentes (1415 años) de España, señala que los alumnos en un primer momento, manifiestan un conjunto de ideas que son indicadoras de un tipo de causalidad mecánica y lineal. Las relaciones ecológicas se interpretan, desde esta óptica, como relaciones de causa- efecto y no se reconoce, por tanto, la interacción, idea esencial para que se produzca la progresión desde una visión simple hacia otra compleja del mundo. Igualmente, los estudiantes manifiestan en general, una visión de la estabilidad y el orden muy rígida y estática, así como una fuerte resistencia a entender que un objeto puede mantener su identidad en transformaciones en las que la conservación no es directamente observable. Según el autor, ello explica el que los alumnos tengan grandes dificultades para entender nociones básicas de la dinámica ecológica como son el equilibrio en los ecosistemas, la sucesión ecológica o el ciclo trófico.

En (1995b), García presento diferentes hipótesis relativas al conocimiento escolar, en el marco de la propuesta curricular, Investigando Nuestro Mundo y se analizaron tres dimensiones o categorías metadisciplinares relativas a la transición desde un pensamiento simple hacia otro complejo, útiles para la caracterización de las ideas de los alumnos en un gradiente de complejidad progresiva y para describir las consiguientes dificultades de aprendizaje y su posible evolución en relación con la instrucción. Posteriormente, propone que una integración de los tres enfoques supone trabajar contenidos relativos a la descripción de elementos y relaciones (primer enfoque), partiendo del estudio de un "ecosistema" muy concreto (segundo enfoque) para después trabajar la idea de ecosistema en general y aplicarla a los diferentes medios (tercer enfoque) (García, \& Rivero, 1996).

En relación con el análisis de las concepciones de los alumnos relativas a las nociones ecológicas, especialmente al concepto de ecosistema (García 2003), desde la perspectiva metadisciplinar aporta tres dimensiones o categorías metadisciplinares referidas a la transición desde un pensamiento simple hacia otro complejo. Estas categorías son: la manera que tienen los sujetos de interpretar la organización del medio, el tipo de relaciones causales que reconocen y la consideración que tienen del cambio y la estabilidad (Garcia, 1995a). 
Según Garcia, (2003), los niveles de progresión para el ecosistema son:

Nivel 1: Donde prima una concepción de ecosistema como Medio Aditivo: Los estudiantes no reconocen una organización subyacente a lo perceptible, en donde las relaciones tróficas a lo sumo si se explicitan son binarias en un medio que es concebido como estático y en donde la diversidad de elementos presentes se quedan en los más evidentes donde las relaciones son sencillas y no configuran un ecosistema.

Desde esta concepción el medio es bien un medio- escenario, es decir es percibido por los alumnos como un fondo homogéneo e indiferenciado donde todo se entremezcla sin una organización aparente, bien un medio aditivo (Astolfi \& Drouin, 1986) en el que éste se entiende como la mera suma de sus componentes. Esta concepción se manifiesta en los alumnos cuando aluden a un inventario de los que hay en él, centrándose únicamente en la enumeración de sus componentes y cuando solo se refieren a algunas relaciones sencillas que se establecen entre éstos (García, \& Rivero, 1996). Esta aditividad dificulta en gran medida la construcción de la noción de red trófica, pues solo se asume un camino posible, de manera que cada elemento se relaciona únicamente con el que le precede o el que va detrás en la cadena (García, 1995a)..

Nivel 2: Ecosistema como Organización Simple: El ecosistema es considerado como un sistema organización simple donde las cadenas tróficas son rígidas en un equilibrio estático. La diversidad de elementos presentes aun continúan siendo las más evidentes en un medio donde las relaciones ecológicas son causales y sencillas.

En este nivel se asume que hay una relación basada en relaciones causales, aunque sean propias de una causalidad mecánica y lineal. También se identifica una mayor diversidad de relaciones ecológicas: relaciones entre la biocenosis y el biotopo, relaciones interespecíficas, así como relaciones intraespecíficas. Además, se reconocen los descomponedores y algunos elementos abióticos.

Nivel 3: Ecosistema como Organización Compleja: El ecosistema es visto como un sistema de organización compleja donde se conciben niveles tróficos dentro de una red trófica dinámica y flexible y se maneja un equilibrio dinámico donde se presenta sucesiones ecológicas, ciclos biogeoquímicos y el concepto de nicho. Las relaciones ecológicas son entendidas como interacciones. En este nivel se reconoce que en el cambio de los ecosistemas se mantiene la organización básica de los mismos.

Según Garcia \& Rivero (1996), la transición desde el modelo en cadena rígida hacia el modelo en red flexible es más bien un proceso complejo en el que se producen avances y retrocesos continuos y en el que la instrucción no garantiza el éxito. En dicha transición los dos obstáculos esenciales parecen ser la falta de una visión descentrada y relativizadora (adopción de la perspectiva propia de cada nivel de la materia) y la concepción aditiva implícita en la "ley del todo o nada".

En esta misma perspectiva de la hipótesis de progresión, Bermúdez \& De Longhi (2006), identifican algunos conceptos ecológicos estructurantes como la biodiversidad, su pérdida y conservación, y las perturbaciones ecosistémicas y proponen su secuenciación curricular, mediante hipótesis de progresión (García, 1997), que contemplan la construcción gradual del saber y suponen distintos niveles de complejidad. En el marco conceptual de la Enseñanza para la comprensión destacan cuatro dimensiones: el contenido, los métodos, los 
propósitos y formas de comunicación. Dentro de cada dimensión, el marco recibe cuatro niveles de comprensión: ingenua, de principiante, de aprendiz y de maestría.

A continuación se presentan las hipótesis de progresión para el tópico Perturbación de acuerdo a los niveles de comprensión formulados por Bermudez \& De Longhi (2006):

Ingenuo. Relacionado con ideas de equilibrio estático, no hay reconocimiento de la existencia de disturbios en los ecosistemas. Se interpreta la inmovilidad como el estado natural y habitual del sistema, presentándose un fuerte paralelismo con las concepciones primarias y simples sobre el equilibrio físico de los cuerpos sólidos (lbarra Murillo \& Gíl Quílez, 2005).

Los desempeños de comprensión ingenua están fundamentados en el conocimiento intuitivo, presentando dificultades para relacionar lo que aprenden en la escuela con su vida cotidiana.

De principiante. Cualquier fuente de variación es considerada una catástrofe (Bermúdez \& De Longhi, 2006a), y no por el hecho de ser poco frecuente (Begon, Harper, \& Townsend, 1995) sino por sus consecuencias desastrosas. Se manifiesta la concepción del "efecto disipador" planteado por White en (1997), según el cual, las consecuencias de una perturbación, situadas en un lugar particular del ecosistema, se debilitan o disipan a medida que se expanden desde una posición particular de la red alimentaria.

De aprendiz. Se reconoce la presencia de disturbios en los ecosistemas asociados, principalmente, a una remoción de biomasa vegetal. Es decir, se tiene conciencia que la acción del ganado, por ejemplo, sobre las pasturas representa una perturbación debido a que es un hecho habitual, no así ciertos fenómenos esporádicos. Los disturbios son una parte constituyente del ambiente, caracterizados por ser regulares, repetidos y esperados. El análisis de los mismos está estrechamente ligado a la escala.

En este nivel de comprensión las personas se basan en conocimientos y modos de pensar disciplinarios, demostrando un uso flexible de conceptos o ideas de la ciencia. En tanto en el siguiente, los desempeños de comprensión de maestría son predominantemente integradores, creativos y críticos, permitiendo la reinterpretación y actuación en el mundo

De maestría. Los disturbios no sólo consisten en una remoción de biomasa, sino que es cualquier evento relativamente discreto en el tiempo que trastorna la estructura de una población, comunidad o ecosistema, cambiando los recursos, la disponibilidad de sustrato o el ambiente físico (Pickett \& White, 1995). La expresión de los disturbios se da en varios niveles de organización jerárquicos (población, comunidad, ecosistema, paisaje).

\section{Consideraciones finales}

-En la mayoría de los trabajos presentados se utilizan principalmente como fuentes de análisis de las concepciones el conocimiento científico, muy pocas investigaciones enfatizan en otras fuentes de conocimiento como los problemas socioambientales. 
-Predomina en el análisis de las concepciones los contenidos conceptuales, pocos trabajos hacen énfasis en los contenidos procedimentales y actitudinales.

-Desde el conocimiento disciplinar, la mayoría de trabajos utilizan como referente teórico de análisis de las concepciones acerca del ecosistema, principalmente las visiones holística y cibernética y no se tienen en cuenta perspectivas más actualizadas como la visión caótica o de no equilibrio.

-En la gran mayoría de trabajos para el análisis de las concepciones sobre el ecosistema no se tiene en cuenta los niveles de complejidad de este contenido en función de los obstáculos de los alumnos.

-Predomina en las diversas investigaciones, el análisis de las concepciones relacionadas con la dinámica del ecosistema y principalmente se abordan las interacciones relacionadas con las redes tróficas, pero no se establece su relación con los flujos de energía, que es una de las propiedades emergentes a nivel de ecosistema.

-Al abordar las redes tróficas no se hace alusión a la vía detritívora sino principalmente a la vía de pastoreo, lo cual incide en la comprensión del papel de los descomponedores principalmente en los flujos de energía y el ciclaje de nutrientes, así como también en tener una visión sistémica y compleja de la dinámica del ecosistema.

-Muy pocos trabajos establecen relaciones entre complejidad, dinámica, estabilidad y diversidad en un ecosistema.

-Respecto a las bases epistemológicas que sustentan las ideas de los alumnos sobre la dinámica del ecosistema, diversos autores señalan que las ideas vitalistas principalmente, aunque también las teleológicas y las antropomórficas, emergen con fuerza en los discursos de los alumnos de básica secundaria

\section{REFERENCIAS BIBLIOGRÁFÍCAS}

Astolfi, J.P. (1988). El aprendizaje de conceptos científicos: aspectos epistemológicos, cognitivos y lingüísticos. Investigación en la Escuela, 23, 65-76.

Astolfi, J.P., \& Drouin, A.M. (1986). Milieu. Aster, 3, 73-110

Astolfi, J.P. (2001). Nivel de formulación Cap 11. En: Conceptos clave en la didáctica de las disciplinas. Serie Fundamentos No 17. Sevilla: Diada Editora.

Adeniyi, E.O. (1985). Misconceptions of selected ecological concepts held by some Nigerian students. Journal of biological Education, 19(4), 311-316.

Barman, C.R., Griffiths, A.K., \& Okebukola, P.A.O. (1995). High school students' concepts regarding food chains and food webs: A multinational study. International Journal of Science Education. 17(6), 775-782.

Bell-Basca, B., Grotzer, T., Donis, K. \& Shaw, S. (2000, April). Using domino and relational causality to analyze ecosystems: realizing what goes around comes around. Paper presented in National Association of Research in science Teaching (NARST). New Orleans, 1- 28.

Begon, M., Harper, J.L., \& Townsend, C. (1995). Ecología: Individuos, poblaciones, comunidades. Barcelona: Ediciones Omega, S.A.

Bermúdez, G., \& De Longhi, A.L. (2006a). Magia y catástrofe en la comprensión ingenua de factores estructuradores de los ecosistemas. Ponencia presentada en 
VII Jornadas Nacionales y II Congreso Internacional de Enseñanza de la Biología "La educación en biología: desafíos y propuestas para una práctica educativa renovadora". ADBiA. 11 a 14 de octubre, Neuquén, Argentina.

Bermúdez, G., \& De Longhi, A.L. (2006). Propuesta curricular de hipótesis de progresión para conceptos estructurantes de ecología. Campo Abierto, 25(2),13-38.

Carlsson, B. (2002). Ecological understanding 2: Transformation - a key to ecological understanding. International Journal of Science Education, 24(7), 701715.

Çetin, G. (2007). English and Turkish pupils' understanding of descomposition. Asia Pacific Forum on Science Learning and Teaching, 8(2), Article 5, 1-24.

Driver R., Squires A., Rushworth, P., \& Wood-Robinson, V. (1994). Nutrition. In Driver R., Squires A., Rushworth, P., \& Wood-Robinson, V. (Eds). Making Sense of Secondary Science: Research into Children's Ideas (pp.27-35). New York: Routledge.

Eilam, B. (2002). Strata of comprehending ecology: Looking through the prism of feeding relations. Science Education, 86 (5), 645-671.

Evagorou, M., Korfiatis, K., Nicolaou, C., \& Constantinou, C. (2009). An Investigation of the Potential of Interactive Simulations for Developing System Thinking Skills in Elementary School: A case study with fifth-graders and sixth-graders. International Journal of Science Education, 31(5), 655-674.

Fernández Manzanal, R., \& Casal Jimenez, M. (1995). La enseñanza de la ecología: Un objetivo de la educación ambiental. Enseñanza de las ciencias, 13(3), 295- 311.

Gallegos, L., Jerezano, M.E., \& Flores, F. (1994). Preconceptions and relations used by children in the construction of food chains. Journal of Research in Science Teaching, 31(3), 259- 272.

García, J.E. (1995a). Epistemología de la Complejidad y enseñanza de la Ecología. El concepto de ecosistema en la escuela secundaria. Tesis doctoral no publicada, Universidad de Sevilla, Sevilla.

García, J.E. (1995b). La transición desde un pensamiento simple hacia un pensamiento complejo en la construcción del conocimiento escolar. Investigación en la Escuela, 27, 7- 20.

Garcia, J. E., \& Rivero A. (1996). La transición desde un pensamiento simple hacia otro complejo en el caso de la construcción de nociones ecológicas. Investigación en la escuela, 28, 23-36.

García, J.E. (1997). Una hipótesis de progresión sobre los modelos de desarrollo en educación ambiental. Investigación en la Escuela, 37, 15-32.

García, J.E. (2003). Investigando el ecosistema. Investigación en la Escuela, 51, 83-100

Gayford, C.G. (1986). Some aspects of the problems of teaching about energy in school biology. European Journal of Science Education, 8(4), 443- 450.

Grajales, A.M., \& González, H.D. (2009). Ecosistemas y pensamiento complejo: una propuesta de intervención para la enseñanza de las ciencias basada en procesos de modelización. Tesis Facultad de educación, Universidad de Antioquia. 
Griffiths, A.K., \& Grant, B. A. C. (1985). High school students' understanding of food webs: Identification of a learning hierarchy and related misconceptions. Journal of Research in Science Teaching, 22(5), 421-436.

Grotzer, T.A. (2002). Causal Patterns in Ecosystem. Lessons to Infuse into Ecosystems Units to Enable Deeper Understanding. Curriculum Module (pp.1-202). Retrieved November 30, 2012, from The Understandings of Consequence Project. Project Zero, Harvard Graduate School of Education, Cambridge, MA. Web site: http://www.pz.harvard.edu/ucp/causalpatternsinscience/pdfs/CausalPatternsInEcosy stems.

Grotzer, T.A., \& Basca, B.B. (2003). How does grasping the underlying causal structures of ecosystems impact students' understanding? Journal of Biological Education, 38(1),16-29.

Grotzer, T.A. (2009). Addressing the Challenges in Understanding Ecosystems: Classroom Studies. Presented at the National Association for Research in Science Teaching (NARST), April 19, 2009, Garden City, CA.

Ibarra Murillo, J., \& Gíl Quílez, M.J. (2005). Enseñar los cambios ecológicos en la secundaria: un reto en la transposición didáctica. Enseñanza de las Ciencias, 23(3), 345-356.

Ibarra Murillo, J. y Gíl Quílez, M.J. (2009). Uso del concepto sucesión ecológica por alumnos de secundaria: la predicción de los cambios en los ecosistemas. Enseñanza de las Ciencias, 27(1), 19-52.

Jacobson, M. J. (2001). Problem solving, cognition, and complex systems: Differences between experts and novices. Complexity, 63, 41-49.

Labbe, C. (2002). Modelisation et conceptualisation L'Exemplle du Cycle du Carbone. Thèse Docteur de L'Universite de la Réunion. Specialite Didactique des Disciplines, Réunion.

Leach, J., Driver, R., Scott, P., \& Wood-Robinson, C. (1991). Progression in conceptual understanding in pupils from age 5 to 16: Cycles of matter, flows energy, interdependency and classification of organisms in ecosystem. A report to the National Curriculum Council. Leeds: Centre for Studies in Science and Mathematics Education. Leeds University.

Leach, J.; Driver, R. ; Scott, P.; Wood-Robinson, C. (1992). Progression in conceptual understanding of ecological concepts by pupils aged 5 to 16 . Centre for Studies in Science and Mathematics Education. Leeds University.

Leach, J.; Driver, R. ; Scott, P.; Wood-Robinson, C . (1996a). Children's ideas about ecology 2: ideas found in children aged 5-16 about the cycling of matter. International Journal of Science Education 18(1), 19-34.

Leach, J., Driver, R., Scott, P. y Wood-Robinson, C. (1996b). Children's ideas about ecology 3: ideas found in children aged 5-16 about the interdependency of organisms. International Journal of Science Education, 18(2),129-141.

Lin $\mathrm{CH}$., \& Hu, R. (2003). Students' understanding of energy flow and matter cycling in the context of the food chain, photosynthesis, and respiration. International Journal of Science Education, 25 (12), 1529-1544.

Magntorn O.(2007). Reading Nature: Developing ecological literacy through teaching. Studies in Science and Technology Education No 6. Submitted to the Faculty of Educational Sciences at Linköping University in partial fulfilment of the 
requirements for the degree of Doctorate of Philosophy. Linköping University. Norrköping, Sweden.

Magntorn, O., \& Hellden, G. (2007). Reading new environments: students' ability to generalize their understanding between different ecosystems. International Journal of Science Education, 29(1), 67-100.

Magro, A.; Simonneaux, L.; Favre, D. y Hemptinne, J.L. (2002). Learning difficulties in ecology. Proceedings of the IV Conference of European Researchers in Didactic of Biology. Toulouse.

Marin, N. \& Cardenas, F.A. (2011). Valoración de los modelos más usados en la enseñanza de las ciencias basados en la analogía "el alumno como científico". Enseñanza de las Ciencias, 29 (1), 35-45.

Peisajovich, B. (2005). El enfoque sistémico: Una propuesta de trabajo para la enseñanza primaria. Correo del Maestro, 113. Consultado el 28 de Noviembre, 2012 ,

de

http://www.correodelmaestro.com/anteriores/2005/octubre/nosotros113.htm

Reiner, M., \& Eilam, B., (2001). Conceptual classroom environment-a system view of learning. International Journal of Science Education, 23(6),551- 568.

Peterfalvi, B., Rumelhard, G., \& Verin, A. (1986). Relations alimentaires. Aster, 3, 111- 189.

Pickett, S.T.A., Parker, V.T., \& Fiedler, P. L. (1992). The new paradigm in ecology: implications for conservation above the species level (pp. 65-88). In P. L. Fiedler. 1992 \& Jain, (Eds). Conservation biology: the theory and practice of nature conservation, preservation and management. New York: Chapman \& Hall.

Pickett, S.T.A. \& Ostfeld, R.S. (1995) The shifting paradigm in ecology.. In: R.L. Knight \& S.F. Bates (Eds), A New Century for Natural Resource Management, (pp. 261-278). Washington: Island Press.

Rincón, M.E., Medellin, F., \& Vargas, C. (2004). Concepciones sobre nociones ecológicas en niños de las escuelas rurales de Villeta (Cundinamarca). Informe CIUP. Bogotá.

Soylu, H. (2006). The effect of gender and reasoning ability on the student' understanding of ecological concepts and attitude towards science. Thesis for the degree of Master of Science, Middle East Technical University, Ankara.

Smith, E. L., \& Anderson, CH. W. (1986, April). Alternative Student Conceptions of Matter Cycling in Ecosystems. Paper presented at the annual meeting of the National Association for Research in Science Teaching, San Francisco, California,.

Westra, R. (2008). Learning and teaching ecosystem behavior in secondary education: Systems thinking and modelling in authentic practices. Unpublished doctoral dissertation, Utrecht University, Nederlands.

White, P. (1997). Naive ecology: Causal judgment about a simple ecosystem. British Journal of Psychology, 88, 219- 233.

White, P. (2000). Naive analysis of food web dynamics: A study of causal judgment about complex physical systems. Cognitive science, 24(4),605- 650. 